\title{
Risk of preterm delivery with increasing depth of excision for cervical intraepithelial neoplasia in England: nested case-control study
}

\author{
(c) (1) (8) OPEN ACCESS
}

\begin{abstract}
Alejandra Castanon epidemiologist ${ }^{1}$, Rebecca Landy statistician ${ }^{1}$, Peter Brocklehurst professor of women's health ${ }^{2}$, Heather Evans consultant in obstetrics and gynaecology ${ }^{3}$, Donald Peebles professor of maternal and foetal medicine ${ }^{2}$, Naveena Singh consultant histopathologist ${ }^{4}$, Patrick Walker consultant in obstetrics and gynaecology ${ }^{3}$, Julietta Patnick director ${ }^{5}$, Peter Sasieni professor of biostatistics and cancer epidemiology ${ }^{1}$, for the PaCT Study Group
\end{abstract}

${ }^{1}$ Centre for Cancer Prevention, Wolfson Institute of Preventive Medicine, Bart's and the London School of Medicine, Queen Mary University of London, London EC1M 6BQ, UK; ${ }^{2}$ Institute for Women's Health, University College London, London, UK; ${ }^{3}$ Department of Gynaecology, Royal Free Hampstead NHS Trust, London, UK; ${ }^{4}$ Division of Cellular Pathology, Barts Health, London, UK; ${ }^{5} \mathrm{NHS}$ Cancer Screening Programmes, Public Health England, Sheffield, UK

\begin{abstract}
Objective To determine the association between depth of excision of cervical intraepithelial neoplasia and risk of preterm birth.

Design Case-control study nested in record linkage cohort study.

Setting 12 hospitals in England.

Participants From a cohort of 11471 women with at least one histological sample taken at colposcopy and a live singleton birth (before or after colposcopy), 1313 women with a preterm birth (20-36 weeks) were identified and frequency matched on maternal age at delivery, parity, and study site to 1313 women with term births (38-42 weeks).

Main outcome measures Risk of preterm birth and very/extreme preterm birth by depth of excisional treatment of the cervical transformation zone.

Results After exclusions, 768 preterm births (cases) and 830 term births after colposcopy remained. The risk of preterm birth was no greater in women with a previous small $(<10 \mathrm{~mm}$ ) excision (absolute risk $7.5 \%$, $95 \%$ confidence interval $6.0 \%$ to $8.9 \%$ ) than in women with a diagnostic punch biopsy $(7.2 \%, 5.9 \%$ to $8.5 \%)$. Women with a medium $(10-14 \mathrm{~mm})$ (absolute risk $9.6 \%$; relative risk $1.28,0.98$ to 1.68$)$, large $(15-19 \mathrm{~mm}$ ) (15.3\%; $2.04,1.41$ to 2.96$)$, or very large ( $\geq 20 \mathrm{~mm}$ ) excision (18.0\%; $2.40,1.53$ to 3.75 ) had a higher risk of preterm delivery than those with small excision. The same pattern was seen in 161 women with very/extremely preterm births (20-31 weeks) and with increasing volume excised. Most births were conceived more than three years after colposcopy, and the risk of preterm delivery did not seem to depend on time from excision to conception.
\end{abstract}

Conclusions The risk of preterm birth is at most minimally affected by a small excision. Larger excisions, particularly over $15 \mathrm{~mm}$ or $2.66 \mathrm{~cm}^{3}$, are associated with a doubling of the risk of both preterm and very preterm births. The risk does not decrease with increasing time from excision to conception. Efforts should be made to excise the entire lesion while preserving as much healthy cervical tissue as possible. Close obstetric monitoring is warranted for women who have large excisions of the cervical transformation zone.

\section{Introduction}

Observational studies and two meta-analyses have found that women treated for cervical intraepithelial neoplasia (CIN) are at increased risk of preterm delivery. ${ }^{1-3}$ The literature suggests an increased risk with greater depth of excision, ${ }^{45}$ but confidence intervals are wide and whether a safe depth exists below which the risk is not increased is unclear.

As part of the NHS cervical screening programme in England, women with abnormal cytology are referred to colposcopy for further investigation. At the first appointment, management can vary from colposcopy only (for low grade cytology and normal colposcopy) to colposcopy plus a diagnostic punch biopsy (for low to moderate cytology with a colposcopic abnormality), and a few women may be treated ("see and treat" for high grade cytology with a defined colposcopic lesion). Women found to have high grade CIN on punch biopsy are treated most commonly by large loop excision of the transformation zone. Rigorous quality assurance in the cervical screening programme 
could explain why performance data for colposcopy and treatment in the NHS are often higher than those found in international studies. ${ }^{67}$

Results from the earlier phase of the study reported here found that compared with the general population, women attending colposcopy had an additional risk of preterm birth of 2.1 per 100 births $(8.8 \%$ compared with $6.7 \%){ }^{8}$ The risk was greater in women who were treated than in those with only a diagnostic punch biopsy, both when the treatment preceded the birth (relative risk $1.19,95 \%$ confidence interval 1.01 to 1.41 ) and when the birth preceded colposcopy $(1.31,0.97$ to 1.76$)$. Therefore, the increased risk in women attending colposcopy may be a consequence of confounding and not caused by treatment. In this phase of the study, we aimed to explore the association between preterm birth and the depth and volume of tissue removed in treatment for cervical disease, the number of excisions carried out before the birth, and the time from treatment to conception.

\section{Methods}

\section{Participants}

We identified women with cervical histology between April 1988 and December 2011 from clinical records in 12 NHS hospitals. They were linked using their NHS number (a unique identifier) and date of birth by Hospital Episode Statistics to hospital obstetric records between April 1998 and March 2011 for the whole of England. Hospital Episode Statistics is a data warehouse containing details of all admissions to NHS hospitals in England. ${ }^{9}$ From Hospital Episode Statistics records, we obtained information on month and year of each delivery, birth weight, whether the birth was a normal vaginal or operative delivery, the mode of onset of labour (spontaneous versus induced), parity, overall index of multiple deprivation at the time of delivery, and any other inpatient diagnoses or operations recorded for the mother.

As obtaining colposcopy and pathology data was the most resource intensive aspect of our pilot study, and many of the women who had attended colposcopy had no births, we designed this study in two phases: the cohort study (phase I) ${ }^{6}$ and the nested case-control study (phase II) reported here. In addition to the births reported in phase I, we identified (using the same methods) 90 from a new site (Worcestershire) and 30 extra births from patients at Hammersmith Hospital (fig $1 \Downarrow$ ).

We divided preterm births (gestational age of 20-36 completed weeks) into moderate (32-36 weeks) and very/extreme preterm (20-31 weeks). ${ }^{10}$ Term births had a gestational age of 38-42 completed weeks. We excluded births at 37 weeks' gestational age to allow a clear divide between term and preterm births. From the cohort, we identified the earliest occurring singleton preterm birth (with any parity) in each woman and frequency matched these to singleton term births in women with no preterm births. Matching was on maternal age at delivery, parity, study site, and whether the birth occurred before or after the first colposcopy to ensure similar characteristics among women with term and preterm births. Only one birth per woman was included in the case-control study.

Hospitals entered colposcopy details into a study database and submitted anonymised pathology reports to Barts Health NHS Trust. Two trained operators entered pathology reports into the study database to ensure that measurements were entered in a standardised way, facilitating the identification of the length, width, and depth of specimens. People searching for and coding colposcopy information were blind to the case-control status of the women.

Extensive data cleaning and checking led the exclusion of one site and of 63 births for which colposcopy records were known to be incomplete (see supplementary methods and fig $1 \downarrow$ ). We also excluded women for whom the only pathology sample reported was non-cervical $(n=18)$ and one woman who was recorded as being sterilised while pregnant.

We excluded women with a diagnosis of cervical cancer at any time $(n=34)$. We also excluded 173 women whose pregnancy was high risk (ICD10 (international classification of diseases, 10th revision) diagnostic codes in supplementary methods and supplementary table A). These diagnoses included diabetes mellitus, hypertension, placenta praevia with haemorrhage, supervision of high risk pregnancy, mental disorders, and diseases of the nervous system complicating pregnancy, childbirth, and the puerperium. We defined excisional treatment as large loop excision of the transformation zone, laser excision, knife cone biopsy, or cone excision not otherwise specified. This paper is restricted to births after colposcopy (188 cases and 209 controls had births before colposcopy).

\section{Statistical methods}

The main exposure of interest was depth of excision before birth, defined as the distance from the distal or external margin to the proximal or internal margin of the excised specimen. ${ }^{11}$ In all participating laboratories, the standard process was to report the depth as the last of three measurements, whereas the reporting of the other two measurements was arbitrary. When the excision was piecemeal, we used the largest fragment depth. For women with more than one excisional treatment, we summed the depths. To assess whether the risk after multiple excisions was greater than that associated with the total depth of tissue excised, we included an indicator for multiple treatments before birth. We did the same analysis to assess the effect of piecemeal excisions. We grouped depth (in $\mathrm{mm}$ ) as $0-9,10-14,15-19, \geq 20$, and unknown depth in accordance with the pre-specified statistical analysis plan. We chose these because they are of practical use and comparable to other cut-offs reported in the literature.

We calculated the volume of the samples assuming treatments were hemi-ellipsoid in shape, using the formula

"volume $=(1 / 2) \times(4 / 3) \times \pi \times($ length $/ 2) \times($ width $/ 2) \times$ depth. ${ }^{.12}$ When only the depth and either length or width were recorded, we assumed the length or width to be the diameter of a circular base. When more than one fragment existed or a woman had multiple treatments, we summed the volumes. Following the statistical analysis plan, we categorised volume by using the 60th and 80th centiles in controls as cut-offs, corresponding to $1.77 \mathrm{~cm}^{3}$ and $2.66 \mathrm{~cm}^{3}$. We developed a risk classification combining depth and volume. We defined time from treatment to conception as time from the last excisional treatment to the date of birth, minus the gestational age at birth.

We calculated relative risks and $95 \%$ confidence intervals by using inverse probability of sampling weighted relative risk regression (a generalised linear model with binomial family and $\log$ link function $)^{13}$ adjusted for maternal age at delivery $(<25$, $25-34,>34$ ), parity (none, one, two, three or more), index of multiple deprivation (in national fifths), and study site. The weights reflect the proportions of term and preterm births from the cohort (phase I) included in the case-control study (see supplementary methods). We estimated absolute risks by the average predicted probability of a preterm birth (averaging over a standardised distribution of confounding variables). We tested 
trends among women who had excisions, excluding those with unknown depth.

In addition to the analysis including all births, to assess sensitivity to the inclusion criteria we re-analysed the data restricting the analyses to preterm births with a spontaneous onset of labour; separating preterm births after excisional treatments that were known to be large loop excisions of the transformation zone from those known to be other excisional procedures; separating preterm births after a piecemeal excision from those with a single piece excised; excluding births with improbable birth weight ( $>3.5 \mathrm{~kg}$ if preterm or $<2.5 \mathrm{~kg}$ if term); excluding women with a diagnosis of CIN2/CIN3 who did not have excisional treatment (as the clinical recommendation is to treat, we may be missing excisions provided in a different clinic); and excluding births after multiple excisional treatments. We used Stata 12 for all analyses.

\section{Results}

From a cohort of 11471 women and 15718 births, we identified 1313 women with a preterm birth and matched them to 1313 women with term births (fig $1 \Downarrow$ ). We obtained full colposcopy details for $87 \%(2284 / 2626)$ of the selected women. After exclusions, 1598 post-colposcopy births (768 preterm and 830 term) were included. Seventy per cent of women had an excisional treatment - most (1005/1101 (91\%) of the women with a single excision before birth) were large loop excision of the transformation zone-and measurements were obtained for $88 \%$ (1193/1360) of excisions. Among the 1114 women with an excision, 301 (27\%) had piecemeal samples and $99(8.9 \%)$ had more than one excisional treatment before the birth.

Of the preterm births, $607(79.0 \%)$ had a gestational age of 32-36 weeks and $161(21.0 \%)$ were very/extreme preterm. Women with a very preterm birth were more likely to have undergone excision than were those with term births $(78 \% \mathrm{v}$ $67 \% ; \mathrm{P}=0.020)($ table $1 \Downarrow)$.

The relative risk of a preterm birth was $38 \%$ greater in women who had an excision than in those who had a punch biopsy (relative risk $1.38,95 \%$ confidence interval 1.10 to 1.72 ). The relative risk of a preterm birth among women who had multiple excisional treatments before the birth $(1.95,1.28$ to 2.95$)$ was greater than the risk among those who had a single excision $(1.34,1.06$ to 1.68$)$. However, after adjustment for (total) depth of excision, the relative risk associated with multiple treatments was 1.14 ( 0.75 to 1.76$)$. This suggests that the excess risk in women with multiple excisions is primarily due to the large amount of tissue removed (that is, the total depth). Therefore, we assigned women with multiple excisional treatments before birth to the appropriate depth category, rather than including them in a separate category. Similarly, the risk of preterm birth in women with piecemeal excisions was non-significantly higher than that predicted by the depth of the largest fragment (relative risk $1.15,0.89$ to 1.49 ), so they are not considered separately.

Among women with excisional treatment, the risk of a preterm birth increased with the depth of excision (table $2 \Downarrow$ ) (P for trend $<0.001)$. The risk of a preterm birth was significantly increased for depths of $15-19 \mathrm{~mm}$ (relative risk 2.04, 1.41 to $2.96)$ and $20 \mathrm{~mm}$ or more (2.40, 1.53 to 3.75$)$ compared with women with small excisions $(<10 \mathrm{~mm})$. The relative risks among women with medium $(1.28,0.98$ to 1.68$)$ or unknown $(1.24$, 0.86 to 1.79 ) excisional depth were intermediate. The absolute risk of a preterm birth was $7.5 \%$ for small excisions, $9.6 \%$ for $10-14 \mathrm{~mm}$ excisions, $15.3 \%$ for $15-19 \mathrm{~mm}$, and $18.0 \%$ for excisions $20 \mathrm{~mm}$ or more deep. The risk of preterm birth in women with a small excision was similar to that among women with a punch biopsy $(7.5 \% \vee 7.2 \%$; relative risk $1.04,0.79$ to 1.37). This result was robust in a variety of sub-analyses (fig $2 \Downarrow)$.

Results restricting the sample to women with spontaneous onset of labour recorded in Hospital Episode Statistics ( $n=1075)$ showed a similar association ( $\mathrm{P}$ for trend $<0.001$ ). The risk of a spontaneous preterm birth was significantly increased for depths of 10-14 mm (relative risk 1.48, 1.09 to 2.01), $15-19 \mathrm{~mm}$ (2.33, 1.52 to 3.58$)$, and $20 \mathrm{~mm}$ or more $(2.27,1.32$ to 3.91$)$ compared with women with small excisions (table $2 \Downarrow$ ).

We found no difference in risk of preterm birth by type of excisional treatment once results were adjusted for depth of excision (relative risk for large loop excision of the transformation zone compared with other excisional treatment $0.94,0.56$ to 1.59 ). We observed a twofold increase in the risk of preterm delivery for excisions that were $15 \mathrm{~mm}$ or greater, for both women treated by large loop excision of the transformation zone and those receiving other excisional treatments (supplementary table B).

We obtained similar results with a somewhat stronger association for all very/extreme preterm births (table $2 \Downarrow$ ). Compared with small excisions, those that were $20 \mathrm{~mm}$ or more deep carried a threefold relative risk $(3.17,1.56$ to 6.47$)$ of a very preterm birth, corresponding to an absolute risk of $6.4 \%$.

Results were robust to a series of sensitivity and sub-analyses. Excluding 59 births with an improbable birth weight or 92 women who had a diagnosis of CIN2/CIN3 but only ever had punch biopsies (fig $2 \Downarrow$ ) did not appreciably alter the results. Considering births after samples that were piecemeal and those that were excised in one piece separately made the relative risk for large/very large excisions slightly smaller but still statistically significant (fig $2 \Downarrow$; supplementary table C). Trends with increasing depth of excision remained significant in all sub-groups and sensitivity analyses (fig $2 \Downarrow$ ).

The strength of association was similar when we considered volume of tissue excised (table $3 \Downarrow$ ). In particular, the relative risk comparing small volume with punch biopsy was 1.03 (0.79 to 1.33 ). We compared total volume of tissue removed with depth of excision to assess whether extra information could be gained by combining these measures (supplementary table D). We observed a trend of increasing risk of preterm delivery with increasing volume in women with medium depth (10-14 mm) of excision $(\mathrm{P}=0.004)$ and a non-significant trend in those with $<10 \mathrm{~mm}$ depth of excision $(\mathrm{P}=0.583)$. We therefore combined volume and depth to classify risk (table $4 \Downarrow$ ). Compared with small depth and volume, excisions deeper than $14 \mathrm{~mm}$ or with a total volume greater than $2.65 \mathrm{~cm}^{3}$ carried double the risk of preterm birth (relative risk 1.97, 1.43 to 2.72).

Among women with large excisions, the risk of preterm birth was not less in those who conceived three years or more after treatment (table $5 \Downarrow$ ). We found no significant excess risk of preterm birth for women with small and medium excisions (table $5 \Downarrow$ ). However, insufficient data were available on women who conceived within six months of small or medium excisions for us to conclude that they are not at increased risk of preterm delivery (relative risk 1.37, 0.74 to 2.53 ).

\section{Discussion}

Although the overall risk associated with excision is minimal, the excess risk seen in women with deeper excisions is substantial. The risk of preterm birth after colposcopy among women who had only a diagnostic punch biopsy and those who had a small $(<10 \mathrm{~mm})$ depth of tissue removed was similar 
(absolute risk 1 in 13). This risk doubled to 1 in 6 when either the depth of the excision or the volume of tissue excised was large. In absolute terms, the risk was $15.3 \%$ in women with large excisions and $7.2 \%$ in those with a punch biopsy before birth compared with $6.7 \%$ in the general population in England. ${ }^{10}$ We found a stronger association for very preterm births: $6.4 \%$ of births in women with very large excisions were very preterm compared with $2 \%$ in those with small excisions and $1.4 \%$ in the general population. ${ }^{14}$ Multiple treatments did not themselves increase the risk of preterm birth; the increased risk in women with multiple excisions seems to be due to the large total depth excised. The increased risk was still apparent three years after treatment and did not seem to change over time.

\section{Strengths and limitations of study}

Owing to the nature of data collection in phase I, some colposcopy dates and procedures were inevitably misclassified. The detailed colposcopy records collected for phase II make such misclassification unlikely. We had very limited information on potential confounding factors such as smoking and ethnicity. Although ethnicity may be related to the risk of having cervical disease, it is unlikely to influence the depth of excision. We have near complete (82\%) smoking information (at the time of colposcopy) for just one of the 12 sites in the study. Analysis of these data suggests no association between smoking and depth of excision, but the numbers are small (143 ever smokers and 86 never smokers) and generalisability is limited. Smoking is unlikely to be able to explain the dose-response relation observed between depth of excision and risk of preterm birth, but we cannot exclude it as a confounder.

Although detailed information on colposcopy was collected by each site and cross referenced with Hospital Episode Statistics records, we had no information on treatments from before 1995 and those at other NHS trusts. Some women in the punch biopsy group may also have received ablative treatment, as only 24 ablative treatments (almost all of which came from one site) were recorded; however, ablation is now rare in England. Before the introduction of large loop excision of the transformation zone in the early 1990s, the preferred treatment was laser ablation (59\% of treatments). ${ }^{15}$ By 1993, after the introduction of national colposcopy guidelines, $61 \%$ of treatments were large loop excision of the transformation zone and only $18 \%$ were laser ablation. ${ }^{15}$ In $2011-12$ just $0.7 \%$ of women had ablation at first colposcopy compared with $44 \%$ having a punch biopsy and $15 \%$ having an excision. ${ }^{16}$ Furthermore, the literature suggests no increased risk of preterm delivery associated with ablative treatment, ${ }^{2}$ so inclusion in the punch biopsy group would not have substantially changed the results.

Our results rely on birth data submitted to Hospital Episode Statistics, and the quality of these data has been questioned. A quarter of births identified were from hospitals that did not participate in our study, showing that we succeeded in identifying such births. However, some (17\%) births in Hospital Episode Statistics do not have gestational age recorded; this could affect the absolute preterm rate. Some high risk pregnancies are likely to have remained in the study, but results were little changed either by inclusion of those we know about or by restricting analysis to births after spontaneous onset of labour. However, Hospital Episode Statistics data are routinely recorded for administrative purposes and are not used as a medical record for management of patients. Misclassification of whether a particular birth was spontaneous will tend to reduce the relative risk but should not affect the excess risk.
The dimensions of the excision were missing for $7 \%$ of participants and were difficult to determine in those with piecemeal excision. However, we saw no indication that using the largest fragment depth when the excision was piecemeal significantly underestimated the effect. A similar problem exists for women treated more than once. Summing the depths of each treatment excludes the possibility of regeneration. It has been suggested that the greater amount of tissue excised the lower the volume regenerated, ${ }^{17}$ but the literature also suggests that women treated more than once are at particularly high risk. ${ }^{7-19}$

\section{Comparison with other studies}

This study has more preterm deliveries with information on size of previous excision $(\mathrm{n}=489)$ than all previous studies combined. A meta-analysis looking at depth included 107 preterm births from three studies, ${ }^{5-20}$ but it did not explicitly consider the trend with increasing depth. ${ }^{2}$ Compared with women who had not attended colposcopy, the relative risk in those with a depth greater than $10 \mathrm{~mm}$ was 2.61 (1.28 to 5.34); it was 1.45 (0.55 to 3.86 ) in those with a depth less than $11 \mathrm{~mm}$. Since this meta-analysis, four further studies (with 318 preterm deliveries) have looked at the depth of excision. ${ }^{4-22}$ The largest found an increased risk of preterm birth associated with treatment of 20 $\mathrm{mm}$ or more compared with less than $10 \mathrm{~mm}$ (odds ratio 1.79 , 1.23 to 2.60$).{ }^{4}$ In keeping with the results from our study, almost all published studies concur that large excisions (depth greater than $15 \mathrm{~mm}$ ) increase the risk of preterm birth. However, ours is the first study to show clinical equivalence in terms of subsequent risk of preterm delivery between small excision $(<10 \mathrm{~mm})$ and punch biopsy. Although we did not use an a priori definition of equivalence in this study, the upper limit of the $95 \%$ confidence interval is 1.37 (for small depth or 1.33 for small volume), compared with 3.86 in the meta-analysis, and none of the subsequent studies included an untreated comparator. NHS colposcopy is carried out by clinicians certified by the British Society for Colposcopy and Cervical Pathology (BSCCP). Most will have trained under the joint BSCCP/Royal College of Obstetrics and Gynaecology programme. This includes a defined number of supervised colposcopies, a module in cytopathology and histopathology, and an exit examination. Three yearly recertification including an audit of activity and confirmation of attendance at a national educational conference is required. Each unit is visited every three years, and the activity and outcomes are examined against defined standards. Colposcopists must see a minimum of 50 women each year. These measures ensure homogenously high standards. This is reflected in three quarters of treated women in the study having a depth of excision less than $15 \mathrm{~mm}$.

We observed no diminution of risk with increasing time from treatment to conception. Literature on this topic is sparse, but one recent paper supports this result. ${ }^{21}$ Although the relative risk of a preterm birth in treated women overall depends on the quality of colposcopy, relative risks associated with increasing size of excision, multiple excisions, and time to conception are generalisable to other colposcopic settings worldwide.

\section{Conclusion}

The risk of preterm birth in women who undergo loop excision of less than $10 \mathrm{~mm}$ in depth and $1.77 \mathrm{~cm}^{3}$ in volume is similar to that in women who have a diagnostic punch biopsy only. Larger excisions, particularly over $15 \mathrm{~mm}$ or $2.66 \mathrm{~cm}^{3}$, are associated with a doubling of the risk of both preterm and very preterm births. Efforts should be made to excise the entire lesion while preserving as much healthy cervical tissue as possible. 
Multiple treatments in themselves did not increase the risk of preterm birth; the increased risk was largely due to the large total depth in women who have had multiple excisions.

Colposcopic treatment guidelines should be updated accordingly.

Contributors: AC coordinated the study with the help of the PaCT Study Group. RL and PS analysed the data and prepared the results. AC, RL, and PS wrote the first draft of the report. PS, AC, PB, PW, HE, NS, and JP participated in the design and establishment of the study. All authors edited the report and approved the final version. PS is the guarantor. Funding: This manuscript presents independent research funded by the National Institute for Health Research (NIHR) under its Research for Patient Benefit (RfPB) Programme (grant reference number PB-PG-1208-16187). The views expressed are those of the author(s) and not necessarily those of the NHS, the NIHR, or the Department of Health. The funder had no input in the design or conduct of the study; the collation, analysis, or interpretation of the data; or the preparation, review, or approval of the manuscript.

Competing interests: All authors have completed the ICMJE uniform disclosure form at www.icmje.org/coi_disclosure.pdf (available on request from the corresponding author) and declare: support for the submitted work as described above; no financial relationships with any organisations that might have an interest in the submitted work in the previous three years; no other relationships or activities that could appear to have influenced the submitted work.

Ethical approval: This study was approved by the Brompton, Harefield, and NHLI research ethics committee, Charing Cross Hospital, London (study reference number: 09/h0708/65).

PaCT Study Group: Pathology report data entry for the study was done by Anna Parberry and Tim Pyke (Barts Health NHS Trust). Members of the PaCT Study Group are responsible for the collection of data included in this study: A Hollingworth and R Wuntakal (Whipps Cross University Hospital London); N Singh and A Parberry (Barts Health NHS Trust); J Palmer (Royal Hallamshire Hospital, Sheffield); N Das, A Andrew, and L Russ (Royal Cornwall Hospital); N Wood and S Preston (Royal Preston Hospital Lancashire); M Hannemann and D Fuller (Royal Devon and Exeter NHS Foundation Trust); K Lincoln, G Wheater, and P Rolland P (The James Cook University Hospital, South Tees); S Ghaem-Maghami and P Soutter (Hammersmith Hospital, Imperial College, London); R Hutson (St James University Hospital, Leeds); P Senguita and J Dent (North Durham County and Darlington Trust); D Lyons (St Mary's Hospital, Imperial College); N Gul, S Edwards, and A Miles (Wirral University Teaching Hospital); S Chin, $\mathrm{H}$ Lenton, and $\mathrm{R}$ Raghavan (Worcestershire Acute Hospitals NHS Trust). All contributors were paid for their contribution to the data collection in this study.

Data sharing: The statistical code is available from the corresponding author at p.sasieni@qmul.ac.uk.

Declaration of transparency: The lead author (study guarantor) affirms that this manuscript is an honest, accurate, and transparent account of the study being reported; that no important aspects of the study have been omitted; and that any discrepancies from the study as planned (and, if relevant, registered) have been explained.
1 Bruinsma FJ, Quinn MA. The risk of preterm birth following treatment for precancerous changes in the cervix: a systematic review and meta-analysis. BJOG 2011:118:1031-41. Kyrgiou M, Koliopoulos G, Martin-Hirsch PL, Arbyn M, Prendiville W, Paraskevaidis E. Obstetric outcome after conservative treatment for intraepithelial or early invasive cervical lesions: systematic review and meta-analysis. Lancet 2006;367:489-98.

3 Noehr B, Jensen A, Frederiksen K, Tabor A, Kjaer SK. Loop electrosurgical excision of the cervix and subsequent risk for spontaneous preterm delivery: a population-based study of singleton deliveries during a 9-year period. Am J Obstet Gynecol 2009;201:33.e1-6.

4 Noehr B, Jensen A, Frederiksen K, Tabor A, Kjaer SK. Depth of cervical cone removed by loop electrosurgical excision procedure and subsequent risk of spontaneous preterm delivery. Obstet Gynecol 2009;114:1232-8.

5 Sadler L, Saftlas A, Wang W, Exeter M, Whittaker J, McCowan L. Treatment for cervical intraepithelial neoplasia and risk of preterm delivery. JAMA 2004;291:2100-6.

6 Albrechtsen S, Rasmussen S, Thoresen S, Irgens LM, Iversen OE. Pregnancy outcome in women before and after cervical conisation: population based cohort study. BMJ 2008;337:a1343.

7 Ortoft G, Henriksen T, Hansen E, Petersen L. After conisation of the cervix, the perinatal mortality as a result of preterm delivery increases in subsequent pregnancy. BJOG 2010;117:258-67.

8 Castanon A, Brocklehurst P, Evans H, Peebles D, Singh N, Walker P, et al. Risk of preterm birth after treatment for cervical intraepithelial neoplasia among women attending colposcopy in England: retrospective-prospective cohort study. BMJ 2012;345:e5174.

9 Health \& Social Care Information Centre. Hospital Episode Statistics. 2013. www.hscic. gov.uk/hes.

10 World Health Organization. Preterm birth. Fact sheet 363. 2012. www.who.int/mediacentre/ factsheets/fs363/en/.

11 Bornstein J, Bentley J, Bosze P, Girardi F, Haefner H, Menton M, et al. 2011 colposcopic terminology of the International Federation for Cervical Pathology and Colposcopy. Obstet Gynecol 2012;120:166-72.

12 Phadnis SV, Atilade A, Young MP, Evans H, Walker PG.. The volume perspective: a comparison of two excisional treatments for cervical intraepithelial neoplasia (laser versus LLETZ). BJOG 2010;117:615-9.

13 Seaman SR, White IR. Review of inverse probability weighting for dealing with missing data. Stat Methods Med Res 2013 Jun;22:278-95.

14 Health \& Social Care Information Centre. NHS maternity statistics - England 2000-2010. www.hscic.gov.uk/searchcatalogue?q=title:\%22nhs+maternity+statistics\%22\&area=\& size $=10$ \&sort $=$ Most + recent.

15 Kitchener HC, Cruickshank ME, Farmery E. The 1993 British Society for Colposcopy and Cervical Pathology/National Coordinating Network United Kingdom Colposcopy Survey: comparison with 1988 and the response to introduction of guidelines. Br J Obstet Gynaecol 1995;102:549-52.

16 Health and Social Care Information Centre. Cervical Screening Programme - England, 2011-2012, 2012. www.hscic.gov.uk/article/2021/Website-Search?productid $=8907$ \& $\mathrm{q}=$ cervical+screening+programme+statistics\&sort=Relevance \&size $=10 \&$ page $=1 \&$ area=both\#top.

17 Papoutsis D, Rodolakis A, Mesogitis S, Sotiropoulou M, Antsaklis A. Appropriate cone dimensions to achieve negative excision margins after large loop excision of transformation zone in the uterine cervix for cervical intraepithelial neoplasia. Gynecol Obstet Invest 2013;75:163-8.

18 Samson S, Bentley JR, Fahey TJ, McKay DJ, Gill GH. The effect of loop electrosurgical excision procedure on future pregnancy outcome. Obstet Gynecol 2005;105:325-32.

19 Jakobsson M, Gissler M, Paavonen J, Tapper AM. Loop electrosurgical excision procedure and the risk for preterm birth. Obstet Gynecol 2009;114:504-10.

20 Raio L, Ghezzi F, Di Naro E, Gomez R, Luscher KP. Duration of pregnancy after carbon dioxide laser conization of the cervix: influence of cone height. Obstet Gynecol 1997;90:978-82.

21 Khalid S, Dimitriou E, Conroy R, Paraskevaidis E, Kyrgiou M, Harrity C, et al. The thickness and volume of LLETZ specimens can predict the relative risk of pregnancy-related morbidity. BJOG 2012;119:685-91.

22 Simoens C, Goffin F, Simon P, Barlow P, Antoine J, Foidart JM, et al. Adverse obstetrical outcomes after treatment of precancerous cervical lesions: a Belgian multicentre study. BJOG 2012;119:1247-55.

Accepted: 15 September 2014

\section{Cite this as: BMJ 2014;349:g6223}

This is an Open Access article distributed in accordance with the Creative Commons Attribution Non Commercial (CC BY-NC 4.0) license, which permits others to distribute, remix, adapt, build upon this work non-commercially, and license their derivative works on different terms, provided the original work is properly cited and the use is non-commercial. See: http://creativecommons.org/licenses/by-nc/4.0/. 


\section{What is already known on this topic}

Most studies of preterm delivery after large loop excision of the transformation zone found that treatment was associated with increased risk

A meta-analysis (including three studies) found an increased risk of preterm birth when the depth of the excised tissue exceeded 10 $\mathrm{mm}$; a large study from Denmark found an increased risk of preterm birth for those with treatments of $\geq 20 \mathrm{~mm}$

The precise role of increasing depth of excision and whether a safe depth exists below which there is no increased risk are unclear

\section{What this study adds}

One in six births in women who had previously had a large $\left(\geq 15 \mathrm{~mm}\right.$ or $\left.\geq 2.66 \mathrm{~cm}^{3}\right)$ excisional procedure at colposcopy were preterm The risk of a preterm birth among women with small procedures at colposcopy was similar to the risk among those not treated at colposcopy before birth

The increased risk in women with multiple excisions seems to be due to the large total depth excised rather than multiple treatments in themselves

\section{Tables}

Table 1/ Main characteristics of women included in study ( $n=1598)$. Values are numbers (percentages) unless stated otherwise

\begin{tabular}{|c|c|c|c|c|}
\hline Characteristics & Term birth (38-43 weeks) $(n=830)$ & $\begin{array}{l}\text { Moderate preterm birth }(32-36 \\
\text { weeks) }(n=607)\end{array}$ & $\begin{array}{l}\text { Very/extreme preterm birth } \\
\quad(20-31 \text { weeks) }(n=161)\end{array}$ & $\begin{array}{l}\text { Absolute risk of preterm } \\
\text { birth (20-36 weeks) }(\%)\end{array}$ \\
\hline \multicolumn{5}{|c|}{ Maternal age at delivery (years) } \\
\hline$<20$ & $2(0.2)$ & $1(0.2)$ & $1(0.6)$ & 9.5 \\
\hline 20 to 24 & $47(5.7)$ & $48(7.9)$ & $15(9)$ & 12.3 \\
\hline 25 to 29 & $204(24.6)$ & $161(26.5)$ & $45(28)$ & 9.6 \\
\hline 30 to 34 & $356(42.9)$ & $244(40.2)$ & $59(37)$ & 8.2 \\
\hline 35 to 39 & $196(23.6)$ & $137(22.6)$ & $36(22)$ & 8.5 \\
\hline$\geq 40$ & $25(3.0)$ & $16(2.6)$ & $5(3)$ & 8.1 \\
\hline \multicolumn{5}{|c|}{ Parity before index birth } \\
\hline None & $423(51.0)$ & $314(51.7)$ & $87(54)$ & 9.0 \\
\hline 1 & $205(24.7)$ & $154(25.4)$ & $36(22)$ & 8.9 \\
\hline 2 & $100(12.0)$ & $67(11.0)$ & $25(16)$ & 8.8 \\
\hline$\geq 3$ & $102(12.3)$ & $72(11.9)$ & $13(8)$ & 8.0 \\
\hline \multicolumn{5}{|c|}{ Worst diagnosis at colposcopy } \\
\hline Normal/benign & $109(13.1)$ & $68(11.2)$ & $17(11)$ & 7.6 \\
\hline Low grade disease $^{\star}$ & $233(28.1)$ & $136(22.4)$ & $34(21)$ & 7.1 \\
\hline High grade disease $\dagger$ & $480(57.8)$ & $394(64.9)$ & $110(68)$ & 9.9 \\
\hline Inadequate/unknown & $8(1.0)$ & $9(1.5)$ & $0(0)$ & 10.6 \\
\hline \multicolumn{5}{|c|}{ Most invasive procedure at colposcopy } \\
\hline Punch biopsy & $274(33.0)$ & $175(28.8)$ & $35(22)$ & 7.4 \\
\hline LLETZ/loop/coneł & $556(67.0)$ & $432(71.2)$ & $126(78)$ & 9.5 \\
\hline
\end{tabular}

*Includes human papilloma virus, cervical intraepithelial neoplasia (CIN) grade 1, and low grade glandular CIN.

tIncludes $\mathrm{CIN}$ grade 2 and 3 , high grade glandular $\mathrm{CIN}$, and ungraded $\mathrm{CIN}$ or glandular $\mathrm{CIN}$.

†Large loop excision of the transformation zone (LLETZ), loop excision, or cone excision. 
Table 2| Relative and absolute risk of preterm birth by depth of excisional treatment in women attending colposcopy before birth

\begin{tabular}{|c|c|c|c|c|}
\hline Procedure at colposcopy & No $(\%)$ cases & No $(\%)$ controls & Relative risk $^{\star}(95 \% \mathrm{Cl})$ & Absolute risk $(95 \% \mathrm{Cl})$ \\
\hline \multicolumn{5}{|l|}{ All preterm births (20-36 weeks) } \\
\hline Punch biopsy before birth & $210(27.3)$ & $274(33.0)$ & 0.96 (0.73 to 1.27$)$ & $7.2(5.9$ to 8.5$)$ \\
\hline \multicolumn{5}{|l|}{ Treatment before birth: } \\
\hline Small excision (1-9 mm deep ) & $173(22.5)$ & $223(26.9)$ & 1 (reference) & 7.5 (6.0 to 8.9$)$ \\
\hline Medium excision (10-14 mm deep) & $182(23.7)$ & $186(22.4)$ & $1.28(0.98$ to 1.68$)$ & $9.6(7.7$ to 11.5$)$ \\
\hline Large excision (15-19 mm deep) & $80(10.4)$ & $48(5.8)$ & 2.04 (1.41 to 2.96$)$ & $15.3(10.5$ to 20.1$)$ \\
\hline Very large excision ( $\geq 20 \mathrm{~mm}$ deep) & $54(7.0)$ & $28(3.4)$ & 2.40 (1.53 to 3.75$)$ & $18.0(10.7$ to 25.1$)$ \\
\hline Unknown treatment depth & $69(9.0)$ & $71(8.6)$ & $1.24(0.86$ to 1.79$)$ & $9.3(6.4$ to 12.2$)$ \\
\hline \multicolumn{5}{|c|}{ Spontaneous preterm births (20-36 weeks) } \\
\hline Punch biopsy before birth & $121(23.7)$ & $274(33.0)$ & 0.84 (0.61 to 1.17$)$ & 4.3 (3.4 to 5.2$)$ \\
\hline \multicolumn{5}{|l|}{ Treatment before birth: } \\
\hline Small excision (1-9 mm deep ) & $117(23.0)$ & $223(26.9)$ & 1 (reference) & $5.1(4.0$ to 6.2$)$ \\
\hline Medium excision (10-14 mm deep) & $137(26.9)$ & $186(22.4)$ & $1.48(1.09$ to 2.01$)$ & 7.5 (5.9 to 9.2$)$ \\
\hline Large excision (15-19 mm deep) & $57(11.2)$ & $48(5.8)$ & 2.33 (1.52 to 3.58$)$ & $11.9(7.6$ to 16.1$)$ \\
\hline Very large excision ( $\geq 20 \mathrm{~mm}$ deep) & $30(5.9)$ & $28(3.4)$ & 2.27 (1.32 to 3.91$)$ & $11.6(5.9$ to 17.2$)$ \\
\hline Unknown treatment depth & $47(9.2)$ & $71(8.6)$ & $1.23(0.81$ to 1.88$)$ & $6.3(4.0$ to 8.5$)$ \\
\hline \multicolumn{5}{|c|}{ All very/extreme preterm births (20-31 weeks) } \\
\hline Punch biopsy before birth & $35(22)$ & $274(33.0)$ & 0.63 (0.38 to 1.06$)$ & $1.3(0.8$ to 1.7$)$ \\
\hline \multicolumn{5}{|l|}{ Treatment before birth: } \\
\hline Small excision (1-9 mm deep ) & $44(27)$ & $223(26.9)$ & 1 (reference) & $2.0(1.3$ to 2.7$)$ \\
\hline Medium excision (10-14 mm deep) & $38(24)$ & $186(22.4)$ & $1.12(0.70$ to 1.82$)$ & $2.3(1.4$ to 3.1$)$ \\
\hline Large excision (15-19 mm deep) & $17(11)$ & $48(5.8)$ & 1.80 (0.91 to 3.54$)$ & $3.6(1.5$ to 5.7$)$ \\
\hline Very large excision ( $\geq 20 \mathrm{~mm}$ deep) & $17(11)$ & $28(3.4)$ & $3.17(1.56$ to 6.47$)$ & $6.4(2.4$ to 10.3$)$ \\
\hline Unknown treatment depth & $10(6)$ & $71(8.6)$ & $0.72(0.34$ to 1.55$)$ & $1.4(0.4$ to 2.4$)$ \\
\hline
\end{tabular}

*Adjusted for parity, index of multiple deprivation, maternal age at delivery, and study site. 
Table 3| Adjusted relative and absolute risk of preterm birth by volume of tissue excised

Volume of excisional treatment No (\%) cases No (\%) controls Relative risk ${ }^{*}(95 \% \mathrm{Cl})$ Absolute risk (\%)

\begin{tabular}{lcccc} 
Punch biopsy & $210(27.3)$ & $274(33.0)$ & $0.97(0.75$ to 1.27$)$ & 7.3 \\
\hline Small volume $\left(\leq 1.77 \mathrm{~cm}^{3}\right)$ & $229(29.8)$ & $297(35.8)$ & 1 (reference) & 7.4 \\
\hline Medium volume $\left(1.78-2.65 \mathrm{~cm}^{3}\right)$ & $95(12.4)$ & $90(10.8)$ & $1.40(1.02$ to 1.93$)$ & 10.4 \\
\hline Large volume $\left(\geq 2.66 \mathrm{~cm}^{3}\right)$ & $165(21.5)$ & $98(11.8)$ & $2.07(1.56$ to 2.76$)$ & 15.4 \\
\hline Unknown volume & $69(9.0)$ & $71(8.6)$ & $1.26(0.88$ to 1.79$)$ & 9.4
\end{tabular}

*Adjusted for parity, index of multiple deprivation, maternal age at delivery, and study site. 
Table 4| Relative risk of preterm birth by risk group in women with treatment before birth

\begin{tabular}{lccc} 
Risk group (largest category for volume or depth) & No (\%) cases & No (\%) controls & Relative risk* (95\% Cl) \\
Small depth AND volume $\left(<10 \mathrm{~mm}\right.$ and $\left.\leq 1.77 \mathrm{~cm}^{3}\right)$ & $142(25.4)$ & $192(34.5)$ & 1 (reference) \\
\hline Medium depth OR volume $\left(10-14 \mathrm{~mm}\right.$ or $\left.1.78-2.65 \mathrm{~cm}^{3}\right)$ & $144(25.8)$ & $170(30.6)$ & 1.15 (0.85 to 1.55$)$ \\
\hline Large depth OR volume $\left(15-20 \mathrm{~mm}\right.$ or $\left.\geq 2.66 \mathrm{~cm}^{3}\right)$ & $149(26.7)$ & $95(17.1)$ & 1.97 (1.43 to 2.72) \\
\hline Very large depth $(\geq 20 \mathrm{~mm})$ & $54(9.7)$ & $28(5.0)$ & $2.44(1.52$ to 3.93$)$ \\
\hline Unknown depth AND volume & $69(12.4)$ & $71(12.8)$ & 1.30 (0.89 to 1.91) \\
\hline
\end{tabular}

*Adjusted for parity, index of multiple deprivation, maternal age at delivery, and study site. 
Table 5| Relative and absolute risk of preterm birth by time from last excisional treatment to conception

Time from last excisional treatment to conception (years) No (\%) cases No (\%) controls Relative risk ${ }^{*}(95 \% \mathrm{Cl})$ Absolute risk (\%) Women with large or very large excision (depth or volume)

\begin{tabular}{lcccc}
\hline 0 to 0.5 & $13(7)$ & $10(8)$ & $0.88(0.35$ to 2.24$)$ & $13.5(1.7$ to 25.3$)$ \\
\hline 0.5 to 1 & $12(6)$ & $13(11)$ & $0.62(0.26$ to 1.47$)$ & $9.4(1.9$ to 17.0$)$ \\
\hline 1 to 3 & $52(26)$ & $27(22)$ & $1.04(0.58$ to 1.84$)$ & $15.8(8.6$ to 23.1$)$ \\
\hline$\geq 3$ & $123(6)$ & $72(59)$ & 1 (reference) & $15.3(11.0$ to 19.5$)$ \\
\hline Women with small, medium, or unknown excision (depth or volume) & $27(8)$ & $25(6)$ & $1.37(0.74$ to 2.53$)$ & $10.2(4.3$ to 16.2$)$ \\
\hline 0 to 0.5 & $28(8)$ & $38(9)$ & $0.84(0.50$ to 1.43$)$ & $6.3(3.2$ to 9.5$)$ \\
\hline 0.5 to 1 & $100(28)$ & $108(25)$ & $1.17(0.84$ to 1.63$)$ & $8.8(6.4$ to 11.1$)$ \\
\hline 1 to 3 & $196(56)$ & $258(60)$ & 1 (reference) & $7.5(6.2$ to 8.9$)$ \\
\hline$\geq 3$ & & &
\end{tabular}

*Adjusted for parity, index of multiple deprivation, maternal age at delivery, and study site. 


\section{Figures}

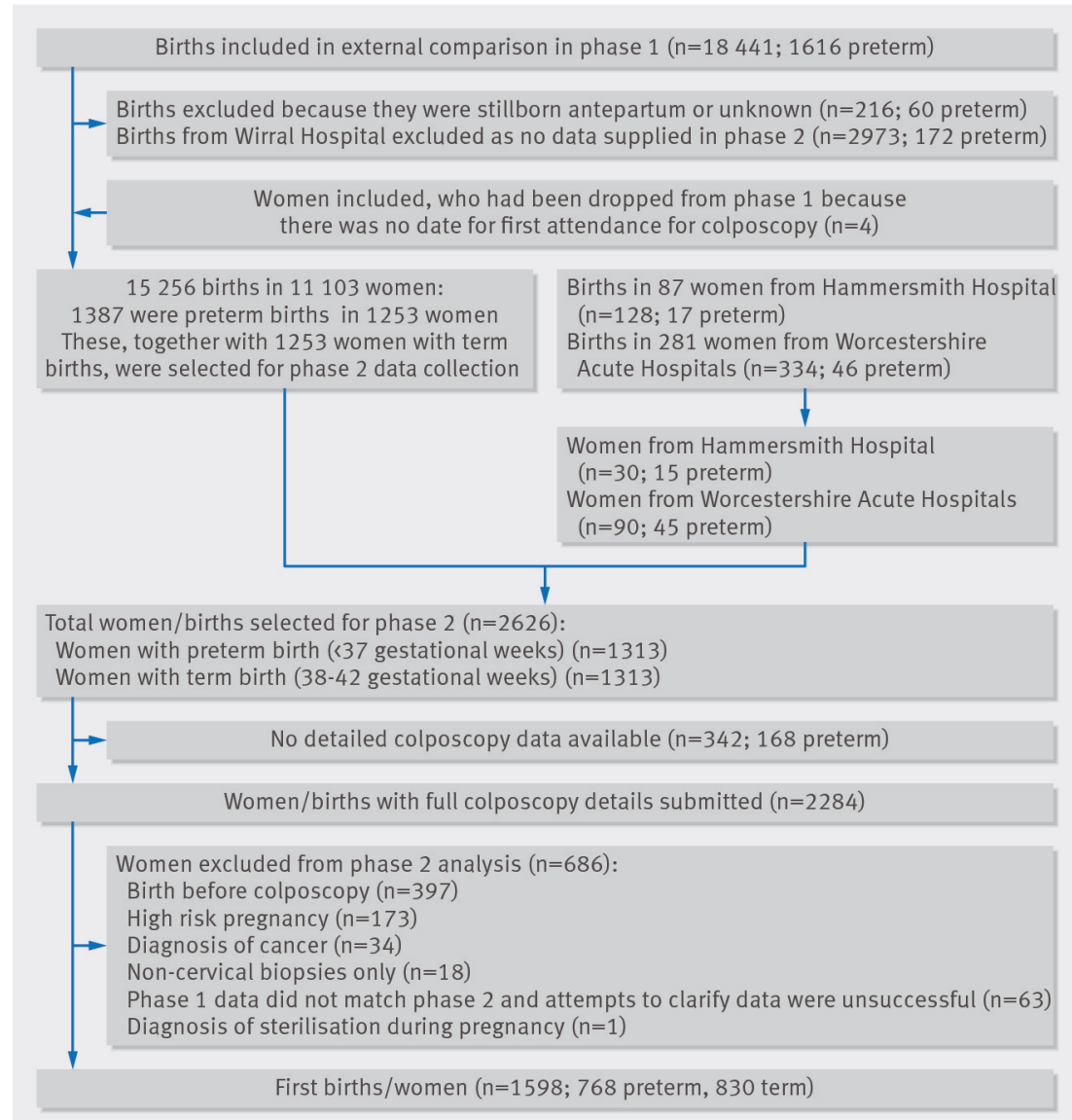

Fig 1 Inclusions in and exclusions from study

All preterm, no exclusions

Sub-analyses

Spontaneous preterm Non-spontaneous preterm

Very preterm birth

Moderate preterm birth

Single piece excision

Piecemeal excision

Treated by LLETZ

Excluding births after multiple treatments Excluding births with unlikely birth weights Excluding punch and diagnosis of high grade

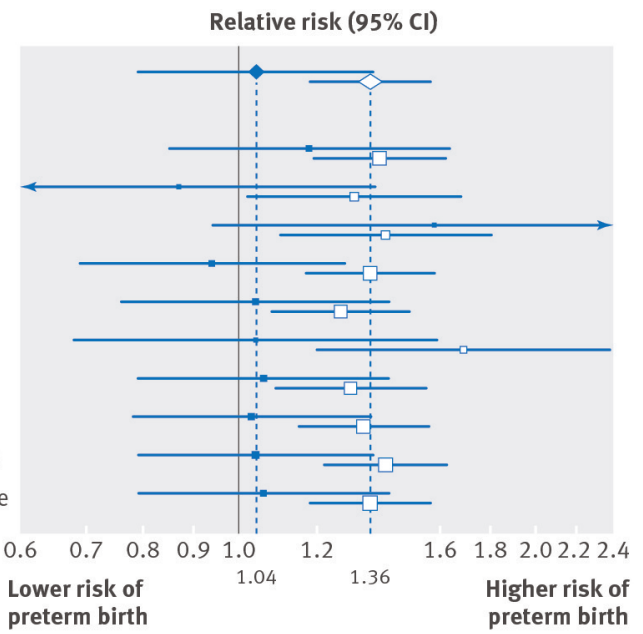

Fig 2 Sensitivity analyses and sub-analyses. Solid squares: relative risk of preterm birth for women with small excisions $(<10 \mathrm{~mm})$ relative to punch biopsy only. Open squares: relative risk of preterm birth with increasing depth of excision, excluding women with unknown depth. LLETZ=large loop excision of the transformation zone 